\title{
Aromatic degradative pathways in Acinetobacter baylyi underlie carbon catabolite repression
}

\author{
Correspondence \\ Ulrike Gerischer \\ ulrike.gerischer@uni-ulm.de
}

Received 17 January 2008

Revised 29 May 2008

Accepted 20 June 2008

\author{
Rita Fischer, Fenja S. Bleichrodt and Ulrike C. Gerischer \\ Institute for Microbiology and Biotechnology, University of Ulm, D-89069 Ulm, Germany
}

\section{INTRODUCTION}

To cope with environmental and metabolic changes bacteria have complex regulatory networks. In their natural environments bacteria are usually exposed to a mixture of carbon sources. To allow the cells to use rapidly metabolizable carbon sources first, a global control mechanism represses the specific induction of other degradative pathways despite the presence of their respective substrates to ensure a hierarchical use of individual carbon sources. This higher level of gene regulation is termed carbon catabolite repression (Stülke \& Hillen, 1999). There is a profound understanding of the molecular mechanism in both enterobacteria and Grampositive bacteria. In both these groups of bacteria, sugars are preferred carbon sources and the phosphotransferase system has a central function in the mechanism. In contrast, in bacteria belonging to the genera Pseudomonas and Acinetobacter, organic acids play an important role as preferred carbon sources and the understanding of the molecular mechanism is far from complete (Gerischer, 2002; Gerischer et al., 2008).

In Acinetobacter baylyi (Vaneechoutte et al., 2006), carbon catabolite repression has been described affecting the expression of the pca-qui operon, the pob operon and the van genes (Dal et al., 2002). These genes code for enzymes

Abbreviations: RLU, relative light units. required for the utilization of protocatechuate $(p c a), p$ hydroxybenzoate $(p o b)$, quinate, shikimate (qui) and vanillate (van). It could be shown that in the presence of the organic acids succinate and acetate in addition to the aromatic compounds the expression level of the pca-qui operon was repressed by at least $90 \%$ during the growth phase. The $p c a$ genes are part of the $\beta$-ketoadipate pathway, which allows the organism to utilize a large variety of aromatic compounds (Williams \& Kay, 2008). This pathway consists of two parallel branches, starting with protocatechuate (3,4-dihydroxybenzoate) or catechol (Cánovas \& Stanier, 1967). The different aromatic substrates are converted to central metabolites via short funnelling pathways (Fig. 1) (Harwood \& Parales, 1996). The expression of the genes required for catechol and protocatechuate degradation, respectively, and that of the genes for the funnelling pathways is regulated independently at the level of specific induction (Gerischer et al., 2008). Most of the genes involved in aromatic catabolism are clustered in two of five islands of catabolic genes on the genome of A. baylyi (Barbe et al., 2004).

Since a strong catabolite repression of the pca operon has been shown ( $\mathrm{Dal}$ et al., 2002; Siehler et al., 2007) we address here the question if other operons linked with aromatic compound catabolism (are, ant, ben, hca, dca) also underlie this regulatory mechanism. To answer this question we produced transcriptional fusions between the catabolic genes and the luc gene, coding for luciferase. 


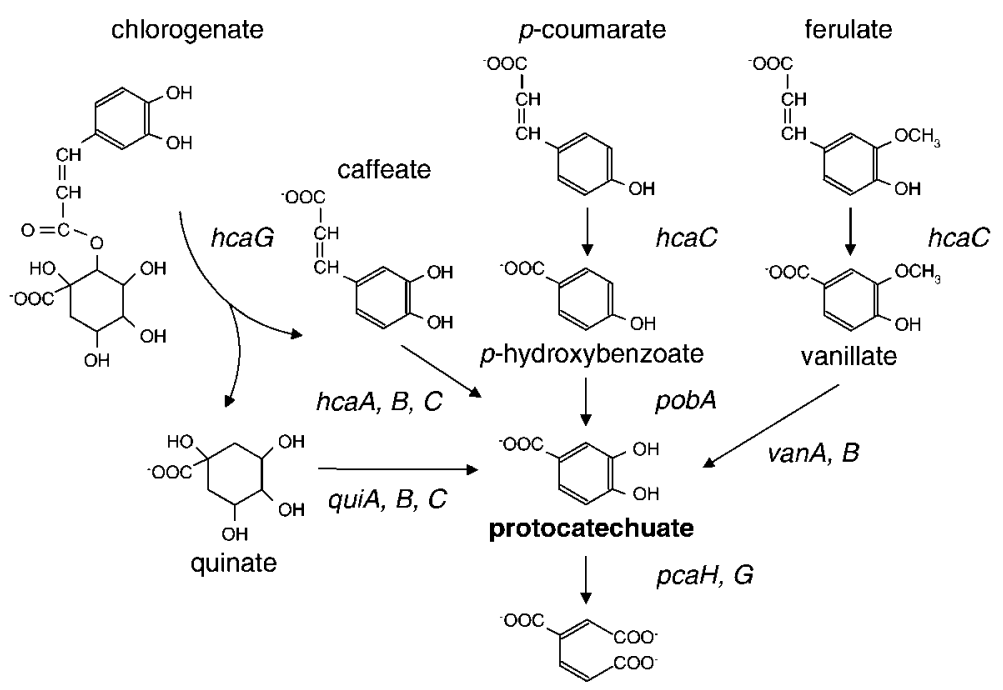

$\beta$-carboxy-cis, cis-muconate

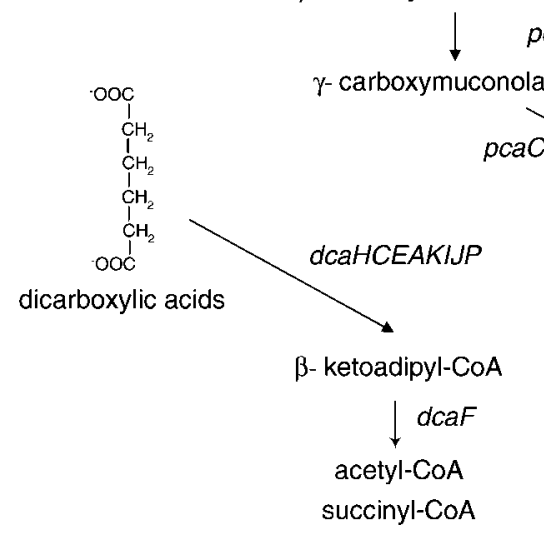

pcaB<smiles>CCC</smiles>

$\beta$ - ketoadipate enol-lactone

pcaD $\downarrow$ catD

$\beta$ - ketoadipate

pcal, $J \downarrow$ catl, $J$

$\beta$ - ketoadipyl-CoA

pcaF $\downarrow$ catF

acetyl-CoA

succinyl-COA

Fig. 1. Catabolic pathways for aromatic compound catabolism in A. baylyi. The degradation of dicarboxylic acids appears to proceed through classic $\beta$-oxidation and converges with the $\beta$-ketoadipate pathway at the level of $\beta$-ketoadipyl-CoA. The names of the respective genes are given next to the arrows.

Using the detected luciferase activity we monitored the gene expression during growth on different carbon sources. We could demonstrate that all five operons underlie strong carbon catabolite repression.

\section{METHODS}

Bacterial strains and growth conditions. Strains of Acinetobacter baylyi (Table 1) were grown on mineral medium at $30{ }^{\circ} \mathrm{C}$ as described earlier (Trautwein \& Gerischer, 2001). The carbon sources were used at the following final concentrations unless indicated otherwise: succinate, $10 \mathrm{mM}$ and $30 \mathrm{mM}$ (indicated in the text); pyruvate, $20 \mathrm{mM}$; lactate, $20 \mathrm{mM}$; acetate, $15 \mathrm{mM}$; succinate plus acetate, $15 \mathrm{mM}$ each. For induction the following concentrations were used: p-hydroxybenzoate, $1 \mathrm{mM}$; benzoate, $1 \mathrm{mM}$; anthranilate, $1 \mathrm{mM}$; benzyl alcohol, $2 \mathrm{mM}$; p-coumarate, $1 \mu \mathrm{M}$; adipate, $1 \mathrm{mM}$. Benzyl alcohol, $p$-coumarate and adipate were dissolved in DMSO.

Antibiotics for A. baylyi strains were used in the following concentrations: spectinomycin, $100 \mu \mathrm{g} \mathrm{ml} \mathrm{m}^{-1}$; ampicillin $150 \mu \mathrm{g}$ $\mathrm{ml}^{-1}$. Escherichia coli strains were grown in $\mathrm{LB}$ medium at $37^{\circ} \mathrm{C}$ supplied with antibiotics when appropriate (spectinomycin, $100 \mu \mathrm{g}$ $\mathrm{ml}^{-1}$, or ampicillin, $100 \mu \mathrm{g} \mathrm{ml}^{-1}$ ). For growth experiments the $A$. baylyi strains with the transcriptional gene fusions were precultured on mineral medium with same carbon source as later used in the experiment except for the aromatic component.

Plasmid and strain construction. Standard methods were used for plasmid isolation, DNA purification, restriction endonuclease cleavage, ligation and transformation (Sambrook \& Russell, 2001). To obtain the DNA region of interest from the A. baylyi genome a PCR using $P f u$-DNA polymerase was performed using the primers listed in Table 2 (benA1/benA2, hcaA1/hcaA2, dcaA1/dcaA2, areA1/areA2, antA1/antA2 for the respective genes). For the construction of pAC116 the PCR fragment containing the ben $A, B$ region was cleaved with HindIII and $K p n \mathrm{I}$ and inserted into the corresponding sites of pUC18. To create plasmid pAC117 the 3254 bp hca region PCR fragment was prepared using NheI and NsiI. To insert the fragment into $\mathrm{pUC18}$, the vector was cleaved by PstI and $\mathrm{XbaI}$ and ligated with the PCR fragment using the compatibility between the PstI/NsiI and $\mathrm{XbaI} /$ NheI ends. The dca PCR fragment was treated with PshAI and $K p n I$ and cloned into pUC18 using HincII and KpnI, yielding pAC118. The plasmid pAC119 was made by cloning a fragment of the are region digested by BstBI and SacI into the pUC18 AccI and SacI sites. To construct plasmid pAC123 the vector pBluescript II SK(+) 
Table 1. Bacterial strains and plasmids used in this study

\begin{tabular}{|c|c|c|}
\hline Strain or plasmid & Relevant characteristics & Source or reference \\
\hline \multicolumn{3}{|l|}{ A. baylyi } \\
\hline ADP1 & Wild-type (strain BD413, ATCC 33305) & Vaneechoutte et al. (2006) \\
\hline ADPU92 & benA-luc transcriptional fusion inserted into benA & This study \\
\hline ADPU93 & $h c a A-l u c$ transcriptional fusion inserted into $h c a A$ & This study \\
\hline ADPU94 & $d c a A-l u c$ transcriptional fusion inserted into $d c a A$ & This study \\
\hline ADPU96 & ant $A$-luc transcriptional fusion inserted into ant $A$ & This study \\
\hline \multicolumn{3}{|l|}{ E. coli } \\
\hline DH5 $\alpha$ & General cloning strain & Hanahan (1983) \\
\hline \multicolumn{3}{|l|}{ Plasmids } \\
\hline pAC116 & 2444 bp fragment containing ben $A, B$ region in pUC18 & This study \\
\hline pAC117 & 3254 bp fragment containing the $h c a A, K$ region in pUC18 & This study \\
\hline $\mathrm{pAC} 121$ & $h c a A-l u c$ transcriptional fusion inserted into pAC117 & This study \\
\hline pAC122 & $d c a A-l u c$ transcriptional fusion inserted into pAC118 & This study \\
\hline pAC123 & 4070 bp fragment containing the ant $A, B$ region in pBluescript II SK $(+)$ & This study \\
\hline pAC124 & areA-luc transcriptional fusion inserted into pAC119 & This study \\
\hline pAC125 & ant $A-l u c$ transcriptional fusion inserted into pAC123 & This study \\
\hline pFW11_luc & Contains the Photinus pyralis luciferase gene & Podbielski et al. (1999) \\
\hline pUC18_luc & luc-aad9 cassette from pFW11_luc cloned into pUC18, with AccI and SmaI & Siehler et al. (2007) \\
\hline
\end{tabular}

was cleaved by BamHI and EcoRV and ligated with the EcoRV/BglIItreated PCR fragment containing the ant region of A. baylyi.

To construct transcriptional fusions between the catabolic genes and the gene for luciferase the luc-aad9 cassette was prepared from pFW11_luc using XhoI and SmaI. The cassette was inserted into pAC117 using the XhoI and SnaBI sites, generating plasmid pAC121, and into pAC118 using the Xho I and StuI sites to create plasmid pAC122. In pAC117 the transcriptional fusion was placed $500 \mathrm{bp}$ from the start of the $h c a A$ gene sequence, creating a 507 bp deletion in this gene. The luc-aad9 cassette in pAC122 was introduced $73 \mathrm{bp}$ from the $d c a A$ gene start, replacing a $69 \mathrm{bp} d c a A$ fragment. For the

Table 2. Oligonucleotides

\begin{tabular}{|ll|}
\hline Primer & \multicolumn{1}{c|}{ Sequence $\left(\mathbf{5}^{\prime} \mathbf{}^{\prime}{ }^{\prime}\right)$} \\
\hline hcaA1 & CGCTTTGAGCAAGCGTAAAC \\
hcaA2 & CAGATTGAGCGCGAACTG \\
dcaA1 & GCCTCTTGTCCAATCAGTGATG \\
dcaA2 & CTTCTTCTGCTGGAACCATGAC \\
dca 3 & TGGCAGGAGTATTCAGGTACAC \\
benA1 & GATCGACTGGTTGGCTAATGC \\
benA2 & GCGGCTTTGGTGAAGCTTTGCTCC \\
ben3 & AAAGCACGGCAGGTACCACACG \\
luc & GTACGTTTAATGGCGGGATCAC \\
areA1 & AAACCGGGAGGTAGATGAGATG \\
areA2 & ATCGGCTGCTAGAGTAAG \\
are3 & ACTCAGTGGCAATGGATG \\
antA1 & CCCTGCAATAGGTGAGATATCG \\
antA2 & ACATCCAGCACCACTTTACG \\
& CCTGATTTGCCTTGCCATTG \\
\hline
\end{tabular}

construction of pAC120 and pAC124, the plasmid pUC18_luc was used. From this plasmid the luc-aad9 cassette could be obtained as a PstI fragment and cloned into pAC116 and pAC119 linearized with NsiI. Plasmid pAC119 has two NsiI recognition sites in close proximity. The cleavage by NsiI created a deletion of $787 \mathrm{bp}$ in the are $A /$ salD gene region and was used to insert the luc-aad9 cassette. The areA-luc fusion is located $445 \mathrm{bp}$ downstream of the transcriptional start of areA. In plasmid pAC116 the NsiI insertion site placed the luc-aad9 cassette $1103 \mathrm{bp}$ downstream from the benA gene start. To create plasmid pAC125, the luc-aad9 cassette was removed from plasmid pFW11_luc using the XmnI and SmaI sites and cloned into pAC123 cut with NruI. The cassette was introduced 88 bp after the ant $A$ gene start. In all cases, the orientation of the cloned fragments was verified by restriction analysis.

For the construction of A. baylyi strain ADPU92 the A. baylyi DNA containing the benA-luc transcriptional fusion was separated from the vector backbone of pAC120 using the DraIII and EcoRI sites. The linear fragment was used for transformation of A. baylyi strain ADP1. A selection for spectinomycin resistance encoded by aad 9 of the lucaad 9 cassette was applied to ensure the homologous recombination of A. baylyi DNA. The other A. baylyi strains containing the transcriptional fusion were made as for strain ADPU92 except for the restriction sites used to remove the insert from the corresponding plasmids. To generate strain ADPU93, plasmid pAC121 was cleaved by BamHI and BglII; to obtain strain ADPU94 the dcaA-luc fusion was cut out from pAC122 by Eco47III and SacI. The plasmid pAC124 was linearized by PmlI and PstI and used to create strain ADPU95. ADPU96 was generated by transformation of A. baylyi with the PmlI$M l u$ I fragment from pAC125.

PCR analysis was employed to verify the correct integration of the constructs into the corresponding location on the chromosome of $A$. baylyi strain ADP1. For this PCR the primer pair comprised one primer hybridizing in the luc-aad 9 cassette (luc primer) and a second primer (ben3, hca3, dca3, are3, antA1) binding in the immediate 
vicinity of the fusion on the chromosome but outside the DNA used for transformation. In all strains the expected PCR fragment could be amplified, thus confirming the desired construction.

Transformation of $\boldsymbol{A}$. baylyi strain ADP1. For transformation of $A$. baylyi, $200 \mu \mathrm{l}$ cell suspension of an overnight culture was transferred in fresh mineral medium supplied with $10 \mathrm{mM}$ succinate and incubated for $2 \mathrm{~h}$ at $30{ }^{\circ} \mathrm{C}$ to allow the cells to start growing again. To $500 \mu \mathrm{l}$ of these cells $0.1-1 \mu \mathrm{g}$ linear DNA was added and incubated at $37{ }^{\circ} \mathrm{C}$ for $3 \mathrm{~h}$ or overnight. Then $100 \mu \mathrm{l}$ volumes of the cells were spread on selective plates and incubated at $30{ }^{\circ} \mathrm{C}$. Grown A. baylyi mutants were transferred on fresh selective plates several times to ensure a pure culture.

PCR. Cells from a bacterial colony diluted in water were used as chromosomal templates. The conditions using $P f u$ DNA polymerase and Taq DNA polymerase were $95{ }^{\circ} \mathrm{C}$ for $3 \mathrm{~min}$, followed by $25-30$ cycles of denaturation at $95{ }^{\circ} \mathrm{C}$ for $1 \mathrm{~min}$, annealing at $53-68{ }^{\circ} \mathrm{C}$ for $1 \mathrm{~min}$ (depending on the primers), and extension at $72{ }^{\circ} \mathrm{C}$ for an appropriate length of time. The primers used are listed in Table 2. For working with $P f u$ DNA polymerase the extension time was doubled.

Determination of luciferase enzyme activity. D-Luciferin was added to a sample of $A$. baylyi cells taken at different times during growth. The luciferase enzyme activity was detected as described earlier (Siehler et al., 2007). The resulting light emission was measured and expressed in relative light units (RLU). The results were normalized by dividing the RLU by the respective $\mathrm{OD}_{600}$ value. The data presented for each culture are taken from mid-exponentialphase cultures and are means based on samples from at least three independent cultures. Error bars indicate standard deviation.

\section{RESULTS}

\section{Construction of $A$. baylyi strains with transcriptional fusions between benA, hcaA, $d c a A$, are $A$ or ant $A$ and the luciferase gene}

To detect the effect of catabolite repression we produced transcriptional fusions between the relevant structural gene promoter and the gene for Photinus pyralis luciferase. For the construction the region of interest was amplified by PCR and introduced into a cloning vector. The luc cassette, containing the luciferase $(l u c)$ gene and a spectinomycinresistance gene, was inserted into the genes benA, hcaA, $d c a A$, are $A$ and ant $A$. The plasmid backbone was separated from the gene construct and the latter was introduced into the chromosome of A. baylyi strain ADP1 via homologous recombination, using the ability for natural transformation of this organism. The newly generated strains contained one copy of the respective transcriptional fusion per cell and thus allowed the determination of gene expression. To examine the expression patterns of the respective promoters we used different carbon sources (lactate, pyruvate, succinate, acetate and a combination of succinate and acetate). The gene expression was induced by adding the specific aromatic compound to the medium. Due to the insertion of the luc cassette the formation of the respective enzyme in each pathway was prevented and further degradation of the respective metabolites was blocked. Therefore the aromatic compounds were added in low concentrations as an inducer. Their concentration was assumed to remain unchanged due to the interrupted catabolism, thus ensuring permanent induction.

\section{Carbon catabolite repression is observed in the ben and ant operons}

Benzoate and anthranilate are independently converted to catechol, allowing further degradation to proceed via the $\beta$ ketoadipate pathway. The ant $A B C$ genes coding for anthranilate dioxygenase (Bundy et al., 1998; Eby et al., 2001) and the ben $A B C$ genes coding for benzoate dioxygenase (Neidle et al., 1991) are homologous. The ben operon is regulated by BenM, a LysR family regulator able to activate transcription synergistically, responding to two effectors (benzoate and muconate) (Bundy et al., 2002; Collier et al., 1998; Ezezika et al., 2007a, b). In studies using a chromosomal antA-lac $Z$ transcriptional fusion it could be shown that the ant $A B C$ expression is induced by anthranilate, but a regulatory protein has not been identified (Bundy et al., 1998). According to these data the ben-luc and ant-luc constructs were induced by adding benzoate or anthranilate, respectively, to the medium.

The expression of the antA gene in strain ADPU96 was investigated during growth on different non-inducing carbon sources with or without the inducer anthranilate (Fig. 2). In all cases the inducing effect of anthranilate (1658 -fold) was obvious. Despite the presence of the same concentration of the inducer in all cultures, there were big differences in expression depending on the nature of the non-inducing carbon source. Growth on a combination of succinate plus acetate and inducer resulted in a repression

(a)

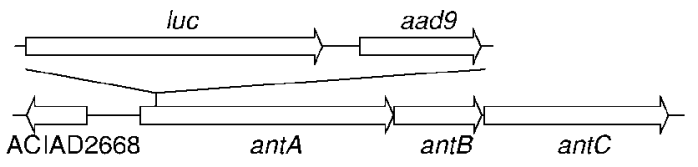

(b)

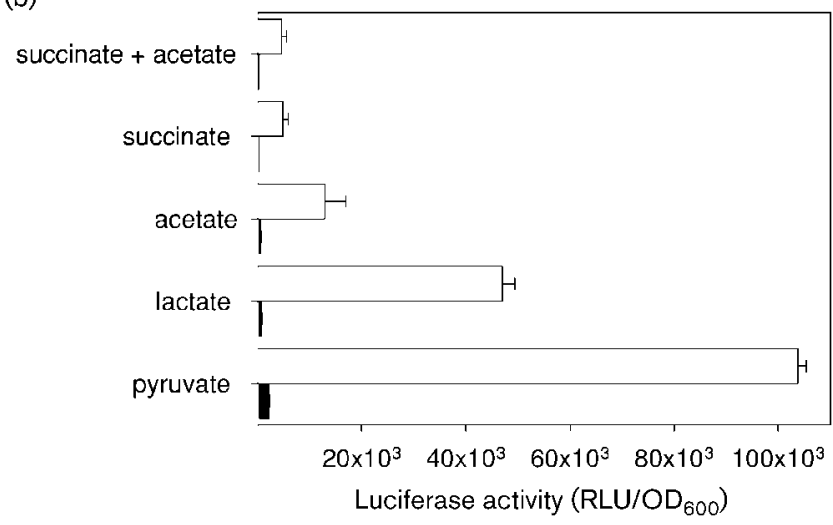

Fig. 2. (a) ant-luc transcriptional fusion in $A$. baylyi strain ADPU96. (b) Luciferase activity of strain ADPU96 measured after growth with the indicated carbon sources in the presence (white bars) or absence (black bars) of $1 \mathrm{mM}$ anthranilate. 
by $95 \%$ as compared to pyruvate. Growth on succinate and inducer had the same repressing effect whereas lactate and acetate each had an intermediate repressing effect. Pyruvate was the carbon source allowing the highest induction.

The strain containing the benA-luc fusion was induced by adding $1 \mathrm{mM}$ benzoate. Using succinate and acetate as a carbon source led to a strong decrease of ben promoter activity in comparison with the least repressing substrate (54-fold, Fig. 3). Succinate and acetate alone caused a light repression (about 2-fold). The highest induction of the ben-luc fusion was observed on lactate. Thus, the ben $A$ promoter showed a similar repression pattern to the ant $A$ promoter, with the slight difference that lactate and not pyruvate was the least repressing carbon source.

\section{Repression of the are operon by additional carbon sources}

The are $A,-B,-C$ and $-R$ genes, encoding enzymes which convert benzyl esters such as benzyl acetate through benzyl alcohol and benzaldehyde to benzoate, are located at one end of the ben-cat cluster (Jones et al., 1999). The are operon is regulated by areR, a XylR-like transcriptional regulator (Jones \& Williams, 2001). For the areA-luc construct the luc cassette was introduced into the areA gene and the transcription was induced by benzyl alcohol. In this strain the utilization of benzyl esters, catalysed by AreA, is blocked but the conversion of benzyl alcohol through benzaldehyde to benzoate followed by conversion to catechol is enabled by AreCB, because the genes are transcribed in the order areCBA. In this case the added

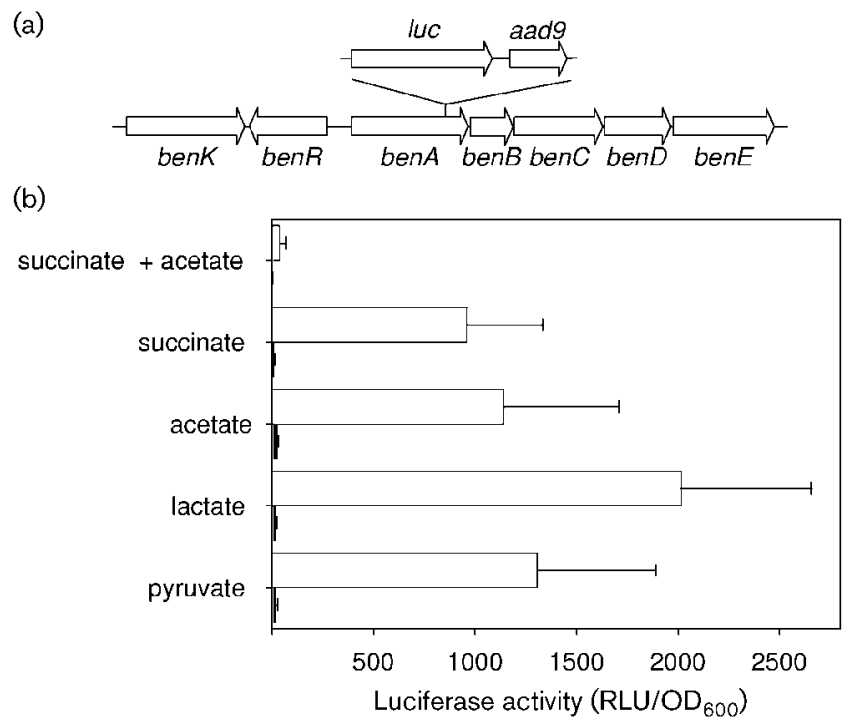

Fig. 3. (a) Map of the ben operon, with the position of the luc cassette in A. baylyi strain ADPU92 indicated. (b) benA promoter activity in strain ADPU92. Luciferase activity was measured after growth with the indicated carbon sources in the presence (white bars) or absence (black bars) of $1 \mathrm{mM}$ benzoate. inducer can be used as a carbon source and was added to the medium at a higher concentration $(2 \mathrm{mM})$.

Using pyruvate in mineral medium supplied with benzyl alcohol led to the highest expression levels (Fig. 4). The strongest reduction of activity was observed after simultaneous addition of succinate and acetate, or succinate alone (reduction by $96 \%$ or $98 \%$, respectively). In summary, all three operons which funnel their substrates into the catechol branch show a transcriptional regulation which strongly depends on the nature of the carbon source(s) present in addition to the substrate inducer. In all cases succinate and acetate were the strongest repressing cosubstrates.

\section{The hca genes are strongly repressed by acetate and succinate}

The Hca enzymes catalyse the conversion of derivatives of 4-hydroxycinnamic acid (chlorogenate, ferulate, caffeate and p-coumarate) into protocatechuate (Smith et al., 2003) (Fig. 1). The $\mathrm{HcaC} \mathrm{CoA}$ ligase initiates metabolism of all these substrates by converting the compounds to the corresponding hydroxycinnamoyl-CoA thioesters. They can act as inducers and relieve the repression caused by the repressor HcaR (a MarR-like regulator). To induce expression of the $h c a$ genes, $p$-coumarate was used. It was added in a low concentration $(1 \mu \mathrm{M})$ because of the known toxic effect caused by the presence of accumulated $p$ coumaroyl-CoA (Parke \& Ornston, 2004).

(a)

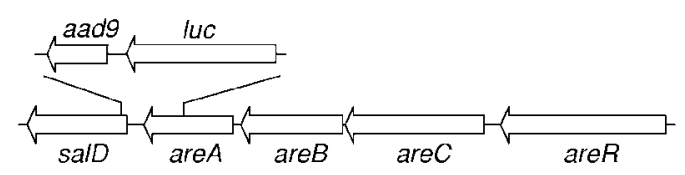

(b)

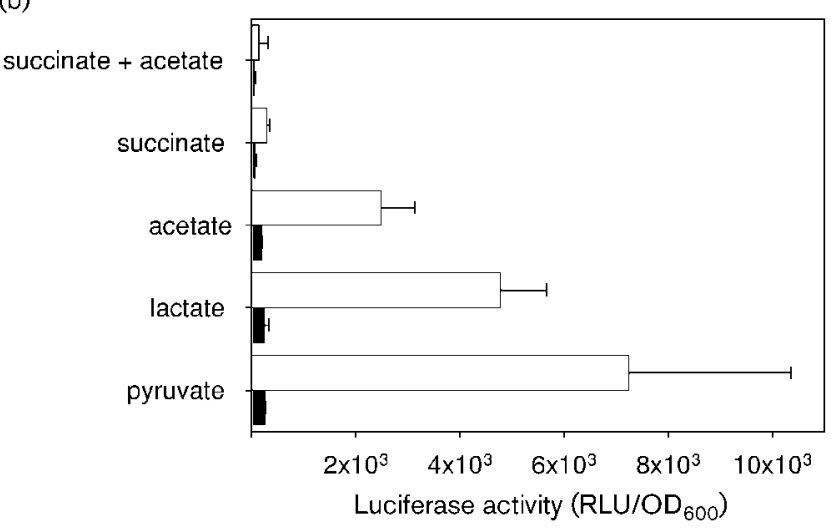

Fig. 4. (a) Map of the are gene region of $A$. baylyi strain ADPU95, showing the insertion position of the luc cassette replacing the indicated $787 \mathrm{bp}$ salD-are $A$ fragment. (b) are operon expression in strain ADPU95. The cells were grown on mineral medium containing the indicated carbon sources in the absence (black bars) or presence (white bars) of $2 \mathrm{mM}$ benzyl alcohol. 
A combination of succinate and acetate in the presence of $p$-coumarate led to a 14-fold reduced luciferase activity in comparison with lactate (Fig. 5). As in strain ADPU92 (benA-luc), lactate as a carbon source resulted in the highest $h c a$ operon expression, followed by pyruvate. Thus the hca operon underlies a strong catabolite repression following the same pattern as observed for other operons connected to the protocatechuate branch of the pathway.

\section{The dca genes underlie catabolite repression}

The dca operon is part of the dca-pca-qui-pob-van-hca supraoperonic gene cluster involved in aromatic compound degradation (Smith et al., 2003). However, the enzymes encoded by the $d c a$ genes are required for the utilization of saturated, straight-chain dicarboxylic acids (Parke et al., 2001). The degradation appears to proceed through classic $\beta$-oxidation. The required enzymes are encoded by two divergent gene clusters, dcaECHF and dcaAKIJP. The dicarboxylic catabolic pathway and the $\beta$ ketoadipate pathway converge at the level of $\beta$-ketoadipylCoA but individual enzymes catalyse for each pathway (an observation that also applies to the $\beta$-ketoadipate pathway itself) (Parke et al., 2001; Young et al., 2005).

The regulator protein of the $d c a$ operon is DcaR, which belongs to the IclR family (Parke et al., 2001). For the luciferase activity assays the $l u c$ cassette was introduced into the $d c a A$ gene. The specific gene expression was

(a)

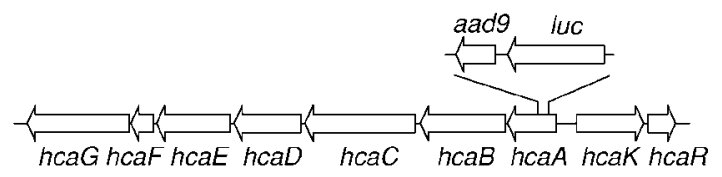

(b)

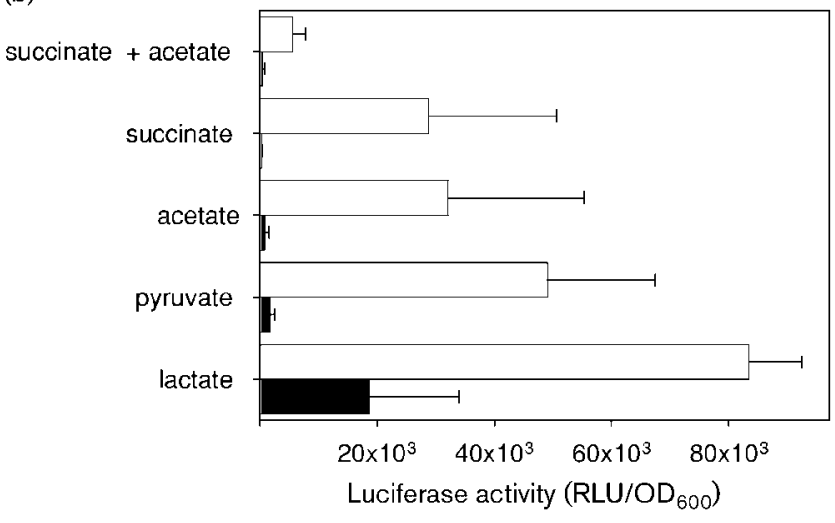

Fig. 5. (a) hca-luc transcriptional fusion in $A$. baylyi strain ADPU93. Insertion of the luc cassette led to a deletion of $507 \mathrm{bp}$ from the hcaA gene. (b) Luciferase activity of strain ADPU93 measured after growth with the indicated carbon sources in the presence (white bars) or absence (black bars) of $1 \mu \mathrm{M} p$ coumarate. induced by adding $1 \mathrm{mM}$ adipate to the medium, which was sufficient since the degradation of the inducer was prevented by the luc gene fusion. As shown in Fig. 6 the activity on succinate plus acetate with adipate decreased by $93 \%$ in comparison to the combination of pyruvate and adipate. Lactate led to a slight repression, and acetate or succinate alone to a stronger repression of $d c a A$ expression. These observations strengthened the evidence that all operons of the dca-pca-qui-pob-van-hca cluster show in principle the same expression pattern in response to the presence of additional carbon sources.

\section{The carbon-source-dependent expression pattern is independent of the induction status}

Looking at the results from the reporter gene studies done with all five operons we find a strong common pattern. All the constructs show the strongest decrease of activity on succinate plus acetate followed by succinate and acetate alone. The highest expression was observed when lactate or pyruvate was used as a carbon source.

This pattern was observed in the presence of the respective substrate inducer as well as in its absence (black bars in Figs 2-6). Thus the differential expression on the noninducing carbon sources investigated here is independent of the induction status, an observation that had been made earlier for the pca-qui operon (Siehler et al., 2007; Trautwein \& Gerischer, 2001).

(a)

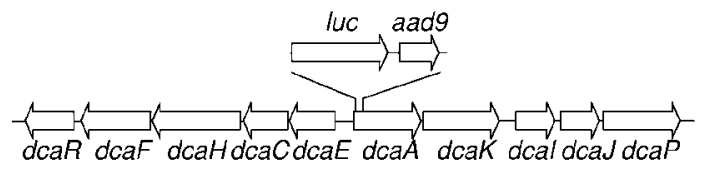

(b)

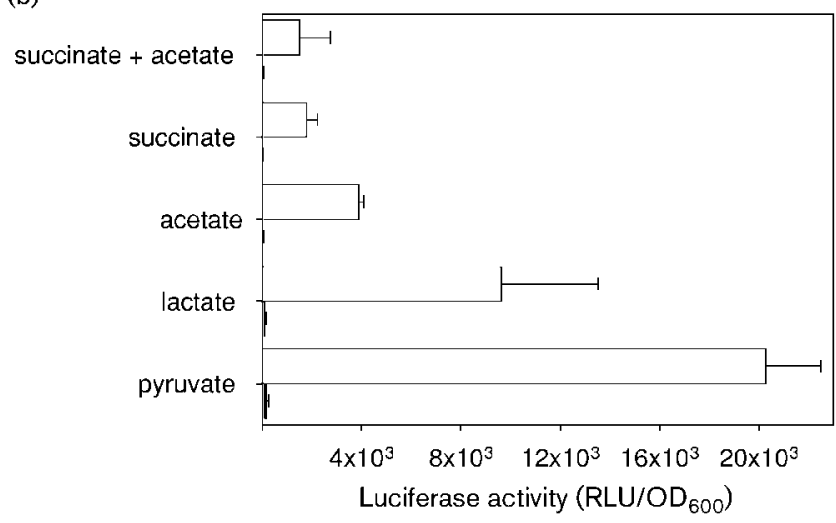

Fig. 6. (a) Map of the dca operon, with the position of the luc cassette in A. baylyi strain ADPU94 indicated. Insertion of the /uc cassette led to the loss of $69 \mathrm{bp}$ from the $d c a A$ gene sequence. (b) Luciferase activity of strain ADPU94. The enzyme activities were measured after growth with the indicated carbon sources in the absence (black bars) or presence (white bars) of $1 \mathrm{mM}$ adipate. 


\section{DISCUSSION}

\section{Strong repression of all operons investigated}

All the operons we investigated showed a strong reduction of promoter activity when the carbon source combination succinate plus acetate in addition to the specific substrate was used (at least $90 \%$, Fig. 7). The respective genes are independent regulatory units and underlie specific regulation by different inducer metabolites and different regulatory proteins. Further, they are located in different regions of the chromosome of $A$. baylyi. These observations are thus in accordance with the phenotype of the global regulatory mechanism carbon catabolite repression.

Acetate is converted into acetyl-CoA (an energy-consuming step), which subsequently feeds into the glyoxylate cycle. Succinate is a metabolite of the citric acid cycle; acetyl-CoA becomes available by the action of malic enzyme forming pyruvate from malate. Both substrates are subsequently oxidized and used for the conservation of energy. Succinate and acetate (or the respective CoA esters) are also the products of the $\beta$-ketoadipate pathway, which in itself does not involve energy conservation. It thus makes sense that these compounds, when present at higher levels, lead to a repression of gene expression in the $\beta$ ketoadipate pathway. Pyruvate and lactate do not - these metabolites are just one or two steps away from acetyl-CoA (catalysed by pyruvate dehydrogenase and lactate dehydrogenase, respectively). It can be speculated that acetate and succinate are abundant substrates in the natural habitats of $A$. baylyi, whereas pyruvate and lactate are not, and therefore no regulatory response has been developed for the latter two. Of course, transport of the substrates into the cells is a process that may also be regulated and therefore also has to be considered. To our knowledge, the

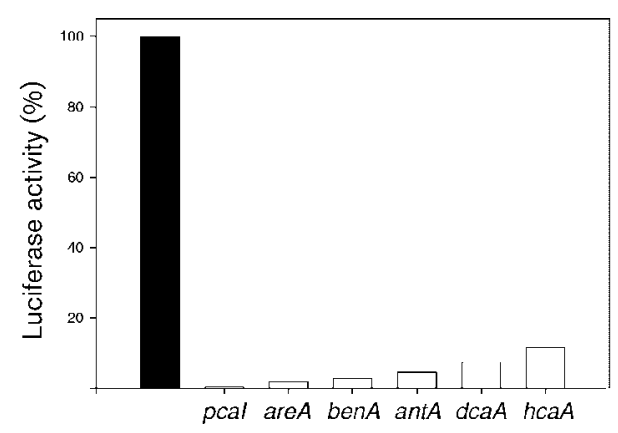

Fig. 7. Extent of repression for the operons investigated. The luciferase activity in all constructed strains on succinate plus acetate (strongest reduction of activity; white bars, target gene indicated) was compared with the corresponding expression on pyruvate (set to $100 \%$, black bar), in the presence of the inducer in all cases. The data for the pcal-luc fusion are derived from measurements of strain ADPU47 growing with or without the inducer $p$-hydroxybenzoate $(1 \mathrm{mM})$. topic of expression/activity of transporters relevant for the substrates concerned in this study has not been invesigated.

\section{Operons affected by the repression in $A$. baylyi}

Repression of catabolic enzymes forming the $\beta$-ketoadipate pathway was described about 40 years ago in Moraxella calcoacetica (Cánovas \& Stanier, 1967), an organism later renamed Acinetobacter calcoaceticus. More recent reports have documented the repression by succinate of many different genes. An A. baylyi strain with additional copies of the ant $A B C$ genes carried on a plasmid lost the ability to use anthranilate as a carbon source (Bundy et al., 1998). The expression of the ant $A B C$ genes in trans in A. baylyi was likely to cause the formation of a higher-than-normal level of catechol during anthranilate catabolism. Catechol probably contributed to the inhibition of growth. The strain grew, however, when succinate was provided together with anthranilate. It was proposed that succinate allows growth by reducing the expression of the ant $A B C$ genes, supporting the findings presented here that succinate is a substrate causing carbon catabolite repression of the ant $A, B, C$ genes.

Further evidence for repression caused by succinate was reported by Jones \& Williams (2001). Benzyl esterase activity on the aromatic compound alone was $33 \%$ higher than in the presence of succinate plus benzyl alcohol. Benzyl alcohol dehydrogenase activity was reduced by $63 \%$ when succinate was provided together with benzyl alcohol as compared to benzyl alcohol alone.

In the $h c a$ operon, a transcriptional hcaE-lacZ fusion was used to detect the promoter activity (Parke \& Ornston, 2004). The detected $\beta$-galactosidase activity at the expense of succinate and $p$-coumarate decreased by $53 \%$ in comparison with the activity measured on $p$-coumarate alone. Using ferulate as substrate in the presence of succinate reduced the activity by $65 \%$.

All these data suggested that multiple operons are repressed in the presence of succinate and an aromatic compound. Our results provide more evidence for this. In terms of quantitative expression differences it has to be kept in mind which method was used. It has been documented that measurements based on luciferase or $\beta$-galactosidase do differ with respect to induction or repression factors, which is due to the lower stability of the luciferase protein (Siehler et al., 2007).

All the operons investigated here are part of two large clusters that each encode pathways for catabolism of plantderived carbon sources of mainly aromatic nature (aresal-ben-cat and dca-pca-qui-pob-hca) (Young et al., 2005).

\section{Clues to the molecular mechanism}

Several lines of evidence indicate an involvement of Cyo (cytochrome $o$ ubiquinol oxidase) in catabolite repression (Dinamarca et al., 2002; Morales et al., 2006; Petruschka 
et al., 2001). Furthermore, evidence for a role of protein $\mathrm{IIA}^{\mathrm{Ntr}}$ (PtsN) in catabolite repression of Pseudomonas putida has been presented (Cases et al., 2001). In some cases evidence has been adduced that the specific regulator protein is the target of catabolite repression. An example is (methyl)phenol degradation in P. putida $\mathrm{H}$ (Müller et al., 1996) or catabolite repression of the alk genes in P. putida GPo1 via downregulation of the level of the AlkS protein (Yuste \& Rojo, 2001). It should be noted that both alkane and phenol degradation pathways are encoded on a plasmid of $P$. putida. Furthermore, transcription requires $\sigma^{54}$ (RpoN)-containing (phl operon) or $\sigma^{\mathrm{s}}$-containing (alk operon) RNA polymerase. Neither of these two conditions applies to the operons under catabolite repression control in A. baylyi (except for the are operon, which depends on $\left.\sigma^{54}\right)$. Nevertheless an involvement of the regulators cannot be ruled out at this point. For PcaU, the activator/repressor protein of the pca-qui operon, it has been documented that the pcaU transcript level corresponds to changes in the amount of the pca-qui transcript levels under all conditions tested. Whether the pcaU transcript level simply is affected by the same mechanism as the pca-qui transcript or is involved in causing the repression is unknown (Siehler et al., 2007)

\section{Crc is the best-studied known player in catabolite repression}

The first potential component of the mechanism described is the Crc (catabolite repression control) protein (MacGregor et al., 1991). Inactivation of the crc gene relieved the repression of a number of genes implicated in the metabolism of carbohydrates (Wolff et al., 1991) and nitrogenated compounds in Pseudomonas aeruginosa and P. putida (Collier et al., 1996; Hester et al., 2000a).

In the $P$. putida OCT plasmid alkane degradation pathway Crc participates in the repression observed when cells grow in rich medium, but it plays no role in the repression caused in mineral medium by organic acids (Yuste \& Rojo, 2001). The number of genes that display an influence by $\mathrm{Crc}$ in their expression has been increased by proteome and RT-PCR analyses to include two of the three aromatic catabolic pathways (Morales et al., 2004).

Crc seems to be involved in post-transcriptional regulation in P. putida (Hester et al., 2000b). In recent reports it could be demonstrated that $\mathrm{Crc}$ can bind specifically to the translation initiation region of two mRNAs for transcriptional regulators (AlkS, BenR), suggesting that Crc modulates gene expression by hindering the access of ribosomes to the ribosome-binding site (Moreno \& Rojo, 2007; Moreno et al., 2007).

A. baylyi contains a Crc homologue. Its inactivation caused withdrawal of catabolite repression upon pca-qui operon expression. In this organism a very strong increase in transcript stability was observed upon $c r c$ deletion for the pca-qui operon (U. Gerischer and others, unpublished data). This may be indicative of different activities of $\mathrm{Crc}$ in different organisms and/or at different genes. It will be exciting to explore Crc function with respect to the $A$. baylyi operons investigated here.

\section{ACKNOWLEDGEMENTS}

We would like to thank Julia Mohr, Maria Trinz, Tina Laux and Olivia Trunk for their contributions. This work was supported by a personal grant (LGFG) to Rita Fischer by the state of BadenWürttemberg, Germany.

\section{REFERENCES}

Barbe, V., Vallenet, D., Fonknechten, N., Kreimeyer, A., Oztas, S., Labarre, L., Cruveiller, S., Robert, C., Duprat, S. \& other authors (2004). Unique features revealed by the genome sequence of Acinetobacter sp. ADP1, a versatile and naturally transformation competent bacterium. Nucleic Acids Res 32, 5766-5779.

Bundy, B. M., Campbell, A. L. \& Neidle, E. L. (1998). Similarities between the ant $A B C$-encoded anthranilate dioxygenase and the ben $A B C$-encoded benzoate dioxygenase of Acinetobacter sp. strain ADP1. J Bacteriol 180, 4466-4474.

Bundy, B. M., Collier, L. S., Hoover, T. R. \& Neidle, E. L. (2002). Synergistic transcriptional activation by one regulatory protein in response to two metabolites. Proc Natl Acad Sci U S A 99, 7693-7698.

Cánovas, J. L. \& Stanier, R. Y. (1967). Regulation of the enzymes of the $\beta$-ketoadipate pathway in Moraxella calcoacetica. 1. General aspects. Eur J Biochem 1, 289-300.

Cases, I., Lopez, J. A., Albar, J. P. \& De Lorenzo, V. (2001). Evidence of multiple regulatory functions for the PtsN (IIA ${ }^{\mathrm{Ntr}}$ ) protein of Pseudomonas putida. J Bacteriol 183, 1032-1037.

Collier, D. N., Hager, P. W. \& Phibbs, P. V., Jr (1996). Catabolite repression control in the pseudomonads. Res Microbiol 147, 551-561.

Collier, L. S., Gaines, G. L. \& Neidle, E. L. (1998). Regulation of benzoate degradation in Acinetobacter sp. strain ADP1 by BenM, a LysR-type transcriptional activator. J Bacteriol 180, 2493-2501.

Dal, S., Steiner, I. \& Gerischer, U. (2002). Multiple operons connected with catabolism of aromatic compounds in Acinetobacter sp. strain ADP1 are under carbon catabolite repression. J $\mathrm{Mol}$ Microbiol Biotechnol 4, 389-404.

Dinamarca, M. A., Ruiz-Manzano, A. \& Rojo, F. (2002). Inactivation of cytochrome $o$ ubiquinol oxidase relieves catabolic repression of the Pseudomonas putida GPol alkane degradation pathway. J Bacteriol 184, 3785-3793.

Eby, D. M., Beharry, Z. M., Coulter, E. D., Kurtz, D. M. \& Neidle, E. L. (2001). Characterization and evolution of anthranilate 1,2-dioxygenase from Acinetobacter sp. strain ADP1. J Bacteriol 183, 109-118.

Ezezika, O. C., Haddad, S., Clark, T. J., Neidle, E. L. \& Momany, C. (2007a). Distinct effector-binding sites enable synergistic transcriptional activation by BenM, a LysR-type regulator. J Mol Biol 367, 616-629.

Ezezika, O. C., Haddad, S., Neidle, E. L. \& Momany, C. (2007b). Oligomerization of BenM, a LysR-type transcriptional regulator: structural basis for the aggregation of proteins in this family. Acta Crystallogr Sect F Struct Biol Cryst Commun 63, 361-368.

Gerischer, U. (2002). Specific and global regulation of genes associated with the degradation of aromatic compounds in bacteria. J Mol Microbiol Biotechnol 4, 111-121. 
Gerischer, U., Jerg, B. \& Fischer, R. (2008). Spotlight on the Acinetobacter baylyi $\beta$-ketoadipate pathway: multiple levels of regulation. In Acinetobacter Molecular Biology, pp. 203-230. Edited by U. Gerischer. Caister Scientific Press.

Hanahan, D. (1983). Studies on transformation of Escherichia coli with plasmids. J Mol Biol 166, 557-580.

Harwood, C. S. \& Parales, R. E. (1996). The $\beta$-ketoadipate pathway and the biology of self-identity. Annu Rev Microbiol 50, 553-590.

Hester, K. L., Lehman, J., Najar, F., Song, L., Roe, B. A., MacGregor, C. H., Hager, P. W., Phibbs, P. V., Jr \& Sokatch, J. R. (2000a). Crc is involved in catabolite repression control of the $b k d$ operons of Pseudomonas putida and Pseudomonas aeruginosa. J Bacteriol 182, 1144-1149.

Hester, K. L., Madhusudhan, K. T. \& Sokatch, J. R. (2000b). Catabolite repression control by $\mathrm{Crc}$ in $2 \times \mathrm{YT}$ medium is mediated by posttranscriptional regulation of $b k d R$ expression in Pseudomonas putida. J Bacteriol 182, 1150-1153.

Jones, R. M. \& Williams, P. A. (2001). areCBA is an operon in Acinetobacter sp. strain ADP1 and is controlled by AreR, a $\sigma^{54}$ dependent regulator. J Bacteriol 183, 405-409.

Jones, R. M., Collier, L. S., Neidle, E. L. \& Williams, P. A. (1999). are $A B C$ genes determine the catabolism of aryl esters in Acinetobacter sp. strain ADP1. J Bacteriol 181, 4568-4575.

MacGregor, C. H., Wolff, J. A., Arora, S. K. \& Phibbs, P. V., Jr (1991). Cloning of a catabolite repression control ( $c r c)$ gene from Pseudomonas aeruginosa, expression of the gene in Escherichia coli, and identification of the gene product in Pseudomonas aeruginosa. J Bacteriol 173, 7204-7212.

Morales, G., Linares, J. F., Beloso, A., Albar, J. P., Martinez, J. L. \& Rojo, F. (2004). The Pseudomonas putida Crc global regulator controls the expression of genes from several chromosomal catabolic pathways for aromatic compounds. J Bacteriol 186, 1337-1344.

Morales, G., Ugidos, A. \& Rojo, F. (2006). Inactivation of the Pseudomonas putida cytochrome $o$ ubiquinol oxidase leads to a significant change in the transcriptome and to increased expression of the CIO and cbb3-1 terminal oxidases. Environ Microbiol 8, 1764-1774.

Moreno, R. \& Rojo, F. (2007). The target for the Pseudomonas putida $\mathrm{Crc}$ global regulator at the benzoate degradation pathway is the BenR transcriptional regulator. J Bacteriol 190, 1539-1545.

Moreno, R., Ruiz-Manzano, A., Yuste, L. \& Rojo, F. (2007). The Pseudomonas putida Crc global regulator is an RNA binding protein that inhibits translation of the AlkS transcriptional regulator. Mol Microbiol 64, 665-675.

Müller, C., Petruschka, L., Cuypers, H., Burchhardt, G. \& Herrmann, H. (1996). Carbon catabolite repression of phenol degradation in Pseudomonas putida is mediated by the inhibition of the activator protein PhlR. J Bacteriol 178, 2030-2036.

Neidle, E. L., Hartnett, C., Ornston, L. N., Bairoch, A., Rekik, M. \& Harayama, S. (1991). Nucleotide sequences of the Acinetobacter calcoaceticus ben $A B C$ genes for benzoate 1,2-dioxygenase reveal evolutionary relationships among multicomponent oxygenases. J Bacteriol 173, 5385-5395.
Parke, D. \& Ornston, L. N. (2004). Toxicity caused by hydroxycinnamoyl-coenzyme A thioester accumulation in mutants of Acinetobacter sp. strain ADP1. Appl Environ Microbiol 70, 2974-2983.

Parke, D., Garcia, M. A. \& Ornston, L. N. (2001). Cloning and genetic characterization of dca genes required for beta-oxidation of straightchain dicarboxylic acids in Acinetobacter sp. strain ADP1. Appl Environ Microbiol 67, 4817-4827.

Petruschka, L., Burchhardt, G., Müller, C., Weihe, C. \& Herrmann, H. (2001). The cyo operon of Pseudomonas putida is involved in carbon catabolite repression of phenol degradation. Mol Genet Genomics 266, 199-206.

Podbielski, A., Woischnik, M., Leonard, B. A. \& Schmidt, K. H. (1999). Characterization of $n r a$, a global negative regulator gene in group A streptococci. Mol Microbiol 31, 1051-1064.

Sambrook, J. \& Russell, D. W. (2001). Molecular Cloning: a Laboratory Manual, 3rd edn. Cold Spring Harbor, NY: Cold Spring Harbor Laboratory.

Siehler, S. Y., Dal, S., Fischer, R., Patz, P. \& Gerischer, U. (2007). Multiple-level regulation of genes for protocatechuate degradation in Acinetobacter baylyi includes cross-regulation. Appl Environ Microbiol 73, 232-242.

Smith, M. A., Weaver, V. B., Young, D. M. \& Ornston, L. N. (2003). Genes for chlorogenate and hydroxycinnamate catabolism ( $h c a)$ are linked to functionally related genes in the dca-pca-qui-pob-hca chromosomal cluster of Acinetobacter sp. strain ADP1. Appl Environ Microbiol 69, 524-532.

Stülke, J. \& Hillen, W. (1999). Carbon catabolite repression in bacteria. Curr Opin Microbiol 2, 195-201.

Trautwein, G. \& Gerischer, U. (2001). Effects exerted by transcriptional regulator PcaU from Acinetobacter sp. strain ADP1. J Bacteriol 183, 873-881.

Vaneechoutte, M., Young, D. M., Ornston, L. N., De Baere, T., Nemec, A., Van Der Reijden, T., Carr, E., Tjernberg, I. \& Dijkshoorn, L. (2006). Naturally transformable Acinetobacter sp. strain ADP1 belongs to the newly described species Acinetobacter baylyi. Appl Environ Microbiol 72, 932-936.

Williams, P. \& Kay, C. M. (2008). The catabolism of aromatic compounds by Acinetobacter. In Acinetobacter Molecular Biology, pp. 99-118. Edited by U. Gerischer. Norfolk, UK: Caister Academic Press.

Wolff, J. A., MacGregor, C. H., Eisenberg, R. C. \& Phibbs, P. V., Jr (1991). Isolation and characterization of catabolite repression control mutants of Pseudomonas aeruginosa PAO. J Bacteriol 173, 4700-4706.

Young, D. M., Parke, D. \& Ornston, L. N. (2005). Oppotunities for genetic investigation afforded by Acinetobacter baylyi, a nutritionally versatile bacterial species that is highly competent for natural transformation. Annu Rev Microbiol 59, 519-551.

Yuste, L. \& Rojo, F. (2001). Role of the $\operatorname{crc}$ gene in catabolic repression of the Pseudomonas putida GPol alkane degradation pathway. $J$ Bacteriol 183, 6197-6206.

Edited by: Michael Hecker 